\title{
How neutral are synonymous codon mutations?
}

Sir - We recently demonstrated the involvement of the muscle-specific calpain in the aetiology of the recessive chromosome 15-linked limb girdle muscular dystrophy (LGMD2A;MIM 253600), including the unexpected observation of at least five distinct calpain mutations in a small consanguinous isolate from the Isle of La Réunion ${ }^{1}$. Additional mutations still segregate in this population, as patients from one family (R15) carry none of the identified mutations. Hence, we systematically screened the calpain gene (CANP3) of a genetically homozygous patient from this family using heteroduplex analyses and sequencing of its various exons ${ }^{2}$. The only genomic sequence variation we identified (Fig. 1a) was an apparently silent third base mutation in glycine codon 624 (GGC $\rightarrow$ GGT) at position 1872 of the cDNA. This synonymous codon could thus be considered as a neutral polymorphism.

It was, however, not until we resorted to illegitimate transcription ${ }^{3}$ that the pathological nature of the mutation could be determined by visualization of an abnormally sized transcription product (Fig. 1b). Sequencing of the corresponding PCR fragment revealed an aberrantly spliced product wherein the last 44 bases of exon 16 are deleted (Fig. 1d), leading presumably to the synthesis of an out of phase translation product (Fig 1c). This mutation, which would therefore be equivalent to a null mutation, is due to gain of a donor splice site resulting from the $\mathrm{C} \rightarrow \mathrm{T}$ transition (M. Fardeau et al., unpublished results). Computer analysis might be helpful in the diagnosis of such mutations. Retrospective analysis using GRAIL ${ }^{4}$

Fig. 1 a, Sequence of CANP3 exon 16 genomic DNA flanking the $\mathrm{C}$ to $\mathrm{T}$ transition (asterisked) from patient R55 (left) a healthy control (right). $b$, Electrophoresis of RT-PCR products of illegitimately transcribed $\mathrm{mRNAs}^{3}$ from lymphoblastoid cells of patient R55 (lane 1) and healthy control (lane 2), lane M; 100 bp ladder (Gibco-BRL). c, Direct sequence analysis of RTPCR product at the junction between exon 16 and 17 in patient R55, demonstrating the loss of the 44 last bases of exon 16. $d$, Normal and new donor CANP3 splicing events and resulting translation products. The arrow represents the site of the $\mathrm{C} \rightarrow \mathrm{T}$ point mutation. $a$

Mutant sequence

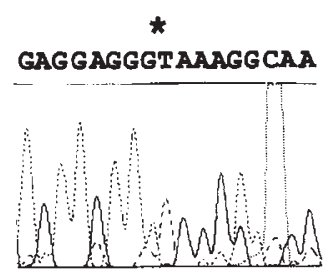

Exon 16

\section{Normal sequence}

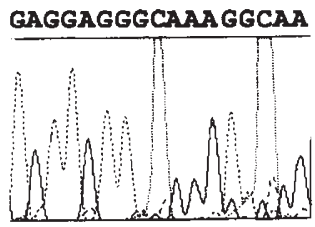

Exon 16
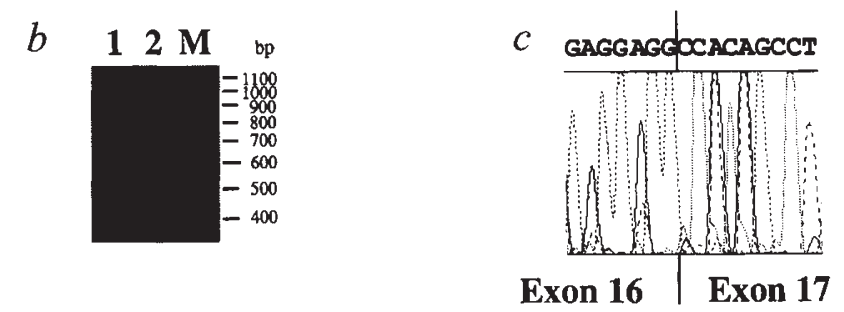

$d$ Nermal (a) and mutant (b) sequences

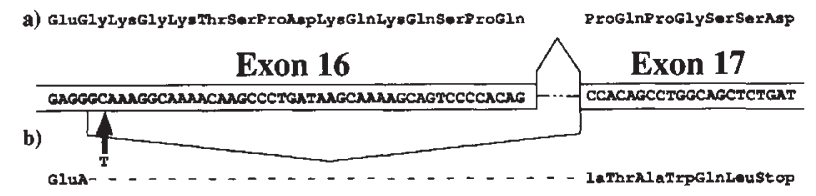

failed, however, to predict the creation of this new splice site, even though the latter had a Senapathy score ${ }^{5}$ similar to the original donor site ( 81 versus 80 ).

The pathogenic nature of a synonymous codon mutation in a Tay-Sachs patient leading to loss of splicing efficiency has been reported ${ }^{6}$. Recently Li et al. demonstrated that a previously described synonymous substitution in the FGFR2 geneled, as suggested $^{7,8}$, to the creation of a donor splice site in the FGFR2 mRNA $^{9}$. Our demonstration of the pathogenic character of a mutation substituting one glycine codon for another underscores the need to interpret such apparently 'neutral' mutations with caution. Until one assesses their impact on mRNA processing and stability or even codon usage bias, it may be difficult to ascertain the neutrality or non-pathogenicity of apparent polymorphisms. In our study, however, this required the testing of illegitimate transcription products as the target gene shows tissue-specific expression in, considering the dystrophic process, an otherwise inaccessible tissue.

\section{Isabelle Richard}

Jacques S. Beckmann'

URA 1922 - Généthon, 1 rue de l'Internationale, 91000 Evry, France ${ }^{1}$ Fondation Jean Dausset - CEPH, 22 rue Juliette Dodu, 75010 Paris, France

\section{Acknowledgements}

Wegratefully acknowledge the expert assistance of L. Brenguier, the cooperation of the Réunion Island families, their clinician M. Fardeau, and the continuous support of the Association Francaise contre les Myopathies.

1. Richard, I. et al. Cell $81,27-40$ (1995)

2. Allamand, V.et al. Am. J. hum. Genet. 56 in the press).

3. Chelly, J. et al. Proc. natn. Acad. Sci. U.S.A. 86, 2617-2621 (1989).

4. Uberbacher, E.C. \& Mural, R. J. Proc. natn Acad. Sci. U.S.A. 88, 11261-11265 (1991).

Shapiro, M. \& Senapathy, P. Nucl. Acids Res. Shapiro, M. \& Senapathy,

6. Akli, J. et al. J. biol. Chem. 265, 7324-7330 (1990).

7eardon, W. et al. Nature Genet. 8, 98-103 (1994)

8. Jabs, E. et al. Nature Genet. 8, 275-279 (1994) 9. Li, X, et al. Nature Genet. 9, 232-233 (1995). 